\title{
Sound Field Calculation for Rectangular Sources
}

\author{
KENNETH B. OCHELTREE, MEMBer, iEeE, ANd LEON A. FRIZZELL SENIOR Member, IEeE
}

\begin{abstract}
A method is presented for calculation of the sound field from a rectangular continuous wave source surrounded by a plane rigid baffle. The approach is illustrated for square sources of $0.5,1,2,5$, 10,20 , and $100 \lambda$ on a side. These results are compared to the sound fields produced by similarly sized circular sources. The beam widths and locations of on-axis minima are similar between the two sources, but the transverse pressure distribution is more uniform in the nearfield of the square source. The effects of attenuation on the sound field of a square source are examined.
\end{abstract}

\section{INTRODUCTION}

$\mathrm{T}$ HE SOUND FIELD produced by a circular piston vibrating in an infinite, plane, rigid baffle has been the subject of many papers and has been well characterized for both continuous wave (CW) [1]-[4] and transient excitation [5], [6]. Since a circular piston has only one descriptive dimension, namely the radius in wavelengths, and axial symmetry is present, the field for a range of sizes of circular sources can be presented in a series of field cross sections. Thus, examples of these theoretically calculated fields are readily available in the literature and a circular source can often be adequately characterized by examining published results.

The fields due to rectangular pistons cannot be characterized as easily since rectangular sources have two descriptive dimensions and their fields lack the axial symmetry associated with circular sources [2], [3], [5], [7]. Thus, the field from the rectangular source is dependent on the ratio of the two sides of the source in addition to their size relative to a wavelength. For any single set of these parameters the lack of axial symmetry means that several cross sections or longitudinal sections are required to illustrate the field. As a result of these additional complexities, field patterns from rectangular sources have not been examined as extensively as those from circular sources.

An efficient field calculation method has been developed which is well suited to the determination of the field from $\mathrm{CW}$ rectangular sources. The fields for several square sources are illustrated and compared with those published for circular sources. In addition, the effects of attenuation in the propagating medium are examined.

Manuscript received February 17, 1988; accepted July 21, 1988. This work was supported in part by Labthermics Technologies, Champaign, IL. in part by a fellowship from The Caterpillar Corp. . Peoria. IL. and in part by NIH Training Grant 5 T 32 CA 09067.

K. B. Ochelrree is with the IBM T. J. Watson Research Center. P.O. Box 704, Yorktown Heights, NY 10598.

L. A. Frizzell is with the Bioacoustics Research Laboratory, Lniversity of Illinois, $1406 \mathrm{~W}$. Green Street, Urbana. IL 61801

IEEE Log Number 8824752.

\section{Methods}

The initial part of this study involved the comparison of several different field calculation methods. These methods included a Fourier transform approach, similar to that developed by Lockwood and Willette [2], and several other methods that divided the radiating surface into incremental areas. The geometry of the incremental areas included points as used by Zemanek [1], circular arcs as suggested by Stepanishen [8], and rectangles [9]. The accuracy of each method was verified through comparison with the point source method. A comparison of calculation times showed that the method employing rectangular areas, the rectangular radiator method, required the least time for a given accuracy. Thus, this method was employed for all field calculations reported here and is discussed in detail below.

The method developed as a part of this study provides the field for an acoustic source, which can be divided into rectangular elements, surrounded by a plane rigid baffle. In the presence of a baffle, the sound pressure amplitude, $p_{0}$, at a point is given by

$$
p_{0}=\frac{j \rho c}{\lambda} \int_{S} u \frac{e^{-(\alpha+j k) r}}{r} d S
$$

where the integration is over the complete radiating surface $S, \rho$ is the density of the medium, $c$ is the phase velocity of the sound waves, $u$ is the velocity amplitude of the piston, $\lambda$ is the wavelength, $k$ is the wave number, $\alpha$ is the attenuation coefficient, and $r$ is the distance between the field point and an elemental area of the piston. This integral has been evaluated by using Huygen's principle and summing the contribution from incremental areas representing the radiating surface [1], [8], [9]. In this study the source was divided into a number of rectangular elements that are too large to be represented as point sources but small enough that simplifying assumptions apply as delineated below.

The total pressure $p_{0}$ at a point in the field is then the sum of the pressure contributed from each element

$$
p_{0}=\frac{j \rho c}{\lambda} \sum_{n=1}^{N} u_{n} \int_{\Delta A} \frac{e^{-(\alpha+j k) r}}{r} d A
$$

where $N$ is the number of elements of size $\Delta A=\Delta h \Delta w$ and $u_{n}$ is the complex surface velocity for element $n$. The complex surface velocity $u_{n}$ is the same for all elements when a uniformly excited rectangular source is considered. For nonuniform excitation, e.g., a phased array 
source, $u_{n}$ is used to represent the local phase and magnitude of the velocity.

The center of element $n$ (subelement $n$ in the case of a multielement transducer) is denoted by $\left(x_{n}, y_{n}\right)$ to simplify the analysis that follows. Additionally, a second coordinate system is defined in $x_{0}, y_{0}$ with its origin centered on the element as shown in Fig. 1. Using these relations in (2) yields

$$
p_{0}=\frac{j \rho c}{\lambda} \sum_{n=1}^{N} u_{n} \int_{-\Delta h / 2}^{\Delta h / 2} \int_{-\Delta w / 2}^{\Delta w / 2} \frac{e^{-(\alpha+j k) r}}{r} d x_{0} d y_{0}
$$

where

$$
r=\sqrt{z^{2}+\left(x-x_{n}-x_{0}\right)^{2}+\left(y-y_{n}-y_{0}\right)^{2}} .
$$

To find an expression for the pressure that is easily evaluated numerically, suitable approximations and their regions of applicability are defined.

By choosing $\Delta h$ and $\Delta w$ to be small, the distance to the field point is much greater than the dimensions of the source and the Fraunhofer approximation can be applied. To simplify application of the Fraunhofer approximation, we define the intermediate variables $x_{n}^{\prime}=x-x_{n}$ and $y_{n}^{\prime}=y-y_{n}$. The Fraunhofer approximation requires that the distance from the origin of the element to the point of interest in the field

$$
\begin{aligned}
R & =\sqrt{z^{2}+\left(x-x_{n}\right)^{2}+\left(y-y_{n}\right)^{2}} \\
& =\sqrt{z^{2}+x_{n}^{\prime 2}+y_{n}^{\prime 2}}
\end{aligned}
$$

to be large compared to $x_{0}$ and $y_{0}$, i.e., $R \gg x_{0}$ and $R \gg y_{0}$. Thus, the exponential term from (3) can be expressed as follows

$$
\begin{aligned}
e^{-(\alpha+j k) r}= & \exp [-(\alpha+j k) \\
& \left.\cdot \sqrt{z^{2}+\left(x_{n}^{\prime}-x_{0}\right)^{2}+\left(y_{n}^{\prime}-y_{0}\right)^{2}}\right] \\
= & \exp [-(\alpha+j k) \\
& \left.\cdot \sqrt{R^{2}-2 x_{n}^{\prime} x_{0}-2 y_{n}^{\prime} y_{0}+x_{0}^{2}+y_{0}^{2}}\right]
\end{aligned}
$$

Using the first two terms of a binomial expansion of the radical on the right side of (6) yields

$$
\begin{aligned}
e^{-(\alpha+j k) r}= & \exp \{-(\alpha+j k) \\
& \left.\cdot\left[R-\frac{x_{n}^{\prime} x_{0}}{R}-\frac{y_{n}^{\prime} y_{0}}{R}+\frac{x_{0}^{2}}{2 R}+\frac{y_{0}^{2}}{2 R}\right]\right\} .
\end{aligned}
$$

For $k x_{0}^{2} / 2 R+k y_{0}^{2} / 2 R$ small compared to $\pi$ and $\alpha<k$, omission of these terms produces a negligible phase error and gives the expression

$$
\begin{aligned}
e^{-(\alpha+j k) r} \simeq & \exp \{-(\alpha+j k) \\
& \left.\cdot\left[R-\frac{x_{n}^{\prime} x_{0}}{R}-\frac{y_{n}^{\prime} y_{0}}{R}\right]\right\} .
\end{aligned}
$$

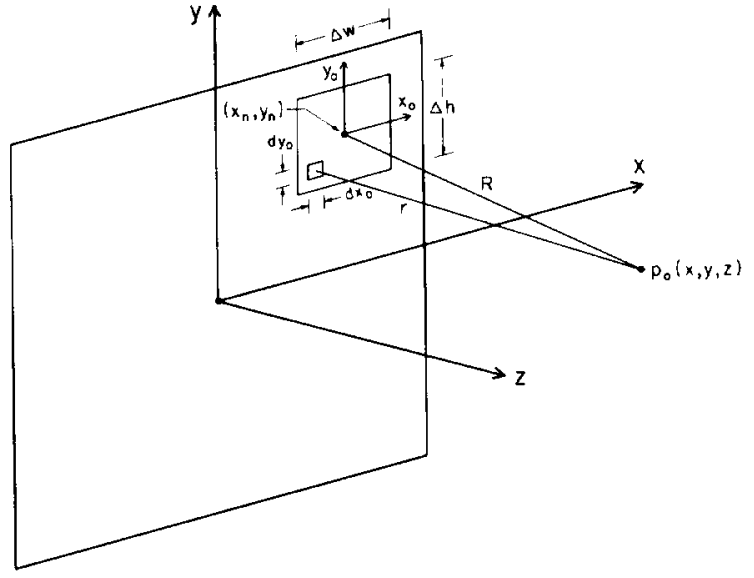

Fig. 1. Coordinate system and geometry used for rectangular radiator

Using this equation, assuming $1 / r \simeq 1 / R$, and substituting into (3) give

$$
\begin{aligned}
p_{0}= & \frac{j \rho c}{\lambda} \sum_{n=1}^{N} \frac{u_{n}}{R} e^{-(\alpha+j k) R} \\
& \cdot \int_{-\Delta w / 2}^{\Delta w / 2} \exp \left[(\alpha+j k) \frac{x_{n}^{\prime} x_{0}}{R}\right] d x_{0} \\
& \cdot \int_{-\Delta h / 2}^{\Delta h / 2} \exp \left[(\alpha+j k) \frac{y_{n}^{\prime} y_{0}}{R}\right] d y_{0} .
\end{aligned}
$$

The assumption that $e^{\alpha x_{n}^{\prime} x_{0} / R} \simeq 1$ for $\Delta w / 2>x_{0}>$ $-\Delta w / 2$ and the equivalent condition for $y_{0}$ are used to reduce the two integrals in (9) to Fourier transform expressions which upon evaluation yield

$$
p_{0}=\frac{j \rho c \Delta A}{\lambda} \sum_{n=1}^{N} \frac{u_{n}}{R} e^{-(\alpha+j k) R} \operatorname{sinc} \frac{k x_{n}^{\prime} \Delta w}{2 R} \operatorname{sinc} \frac{k y_{n}^{\prime} \Delta h}{2 R}
$$

The relation in (10) is a straightforward summation of complex terms, representing the pressure due to a rectangular source, but is only useful if the approximations used are applicable. The three assumptions used to derive (10) are that $k x_{0}^{2} / 2 R+k y_{0}^{2} / 2 R$ is small compared to $\pi$, $1 / r \simeq 1 / R$, and $e^{\alpha x_{n}{ }_{n} x_{0} / R} \simeq 1$. Since the maximum absolute values of $x_{0}$ and $y_{0}$ are $\Delta w / 2$ and $\Delta h / 2$, respectively, these conditions can be expressed as

$$
\begin{aligned}
& \text { 1) } \cos \left(k \Delta w^{2} / 8 R+k \Delta h^{2} / 8 R\right)=1 \\
& \text { 2) } r / R \simeq 1
\end{aligned}
$$

and

$$
\text { 3) } e^{\alpha\left(x_{n}^{\prime} \Delta w+y_{n}^{\prime} \Delta h\right) / 2 R}=1 \text {. }
$$

These conditions provide limitations on the sizes of $\Delta w$ and $\Delta h$ that can be used for computation. The first con- 
dition provides the limitation on $\Delta w$ that $\pi \gg$ $k \Delta w^{2} / 8 R$. This condition can be rewritten as $R>$ $\Delta w^{2} / 4 \lambda$, which is equivalent to the provision that the field point be in the farfield for a source of size $\Delta w$. Since $z$ is less than $R$, an equivalent condition is $z \gg \Delta w^{2} / 4 \lambda$. A constant $F$ can be defined such that the first condition is given by the relation

$$
\Delta w \leq \sqrt{\frac{4 \lambda z}{F}} .
$$

The constant $F$ represents the distance from the source to the field point relative to the distance to the nearfield-farfield transition for a source of size $\Delta w(F=10$ for all field calculations reported here). The inequality sign in (11) must be retained so that the width of the source can be made an integer multiple of $\Delta w$. A similar relation applies to the selection of $\Delta h$.

The use of (11) for determining $\Delta w$ and $\Delta h$ ensures that conditions 2) and 3) are satisfied. Also, significant cancellation of errors occurs from the contribution to the field from one edge of an element to the other, so that actual errors are less than would be predicted by a first order approximation. Thus, a second-order analysis would be required to establish an upper bound on the error.

The expression in (10) was implemented on a VAX 11/ 730 computer using (11) to specify the values of $\Delta w$ and $\Delta h$. The relationship between the size of the element and $z$, given by (11), was used to minimize computation time by reducing the number of elements, $N$, as the distance from the array increased, while preserving the same degree of accuracy.

\section{Results AND Discussion}

The rectangular radiator method was used to calculate the acoustic field in a lossless medium for square sources ranging in size from 0.5 to $100 \lambda$ on a side. The pressure fields were calculated for longitudinal sections in the $y=$ 0 plane with the field normalized at each axial distance (see Fig. 1 for geometry). The results are shown in Figs. 2-8 using contour plots of the $-3-$ and $-6-\mathrm{dB}$ levels. Since the field is normalized at each axial distance, these plots show the $-3-$ and $-6-\mathrm{dB}$ beam width versus distance from the source and illustrate the multiple maxima that occur close to the source. Included with these results are plots of the normalized axial intensity. Axial distances are relative to the nearfield-farfield transition distance ( $s^{2} / 4 \lambda$, where $s$ is the length of a side of the source) to remove frequency dependence and to aid in comparison with the results for a circular source as computed by $\mathrm{Ze}$ manek [1].

The normalized field approaches a limiting pattern as the source size becomes much larger than a wavelength, as observed for a circular source [1]. As a single cross
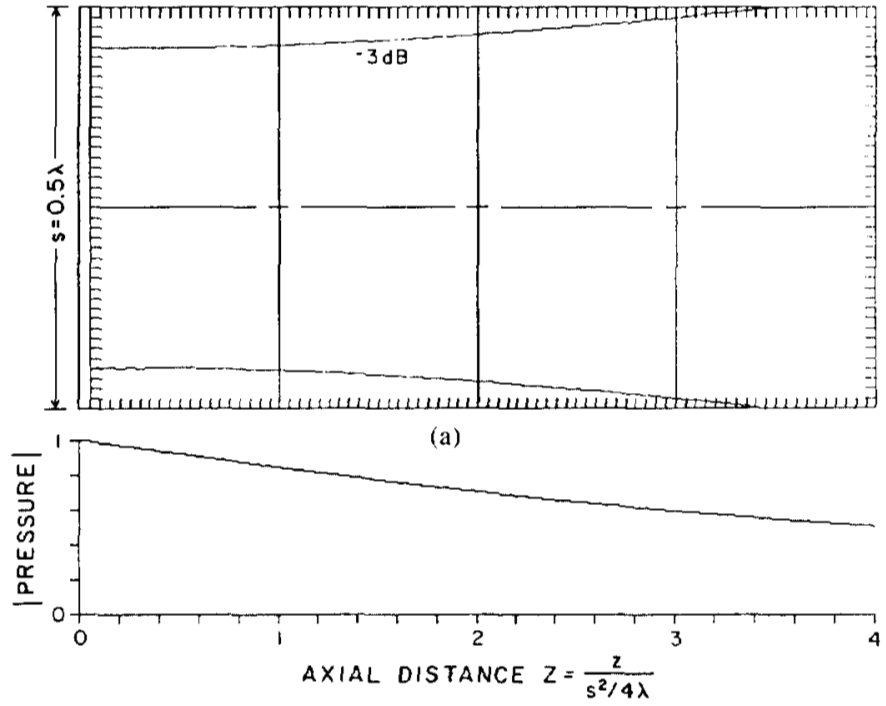

(b)

Fig. 2. Square source with sides equal to $0.5 \lambda$. (a) Sound pressure contour normalized at each axial distance. (b) Normalized magnitude of on-axis pressure.
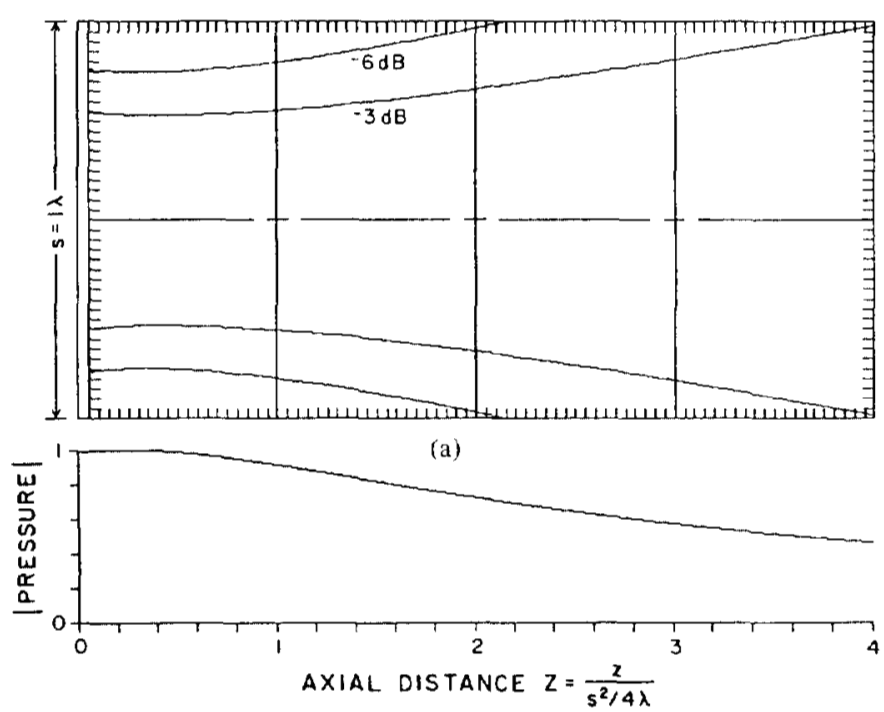

(b)

Fig. 3. Square source with sides equal to $1 \lambda$. (a) Sound pressure contour normalized at each axial distance. (b) Normalized magnitude of on-axis pressure.

section is not completely sufficient to characterize the field for a square source, a diagonal section $(x=y)$ through the field produced by a two-wavelength square source is shown in Fig. 9. The field for this diagonal section is proportionally broader but otherwise does not vary significantly from the $y=0$ section shown in Fig. 4 .

Sound pressure contours for a circular source, as produced by Zemanek [1], are shown in Fig. 10. The differences between the fields for a circular source and a square source are evident. The field of a square source lacks the on-axis nulls and lateral variations that occur in the field 


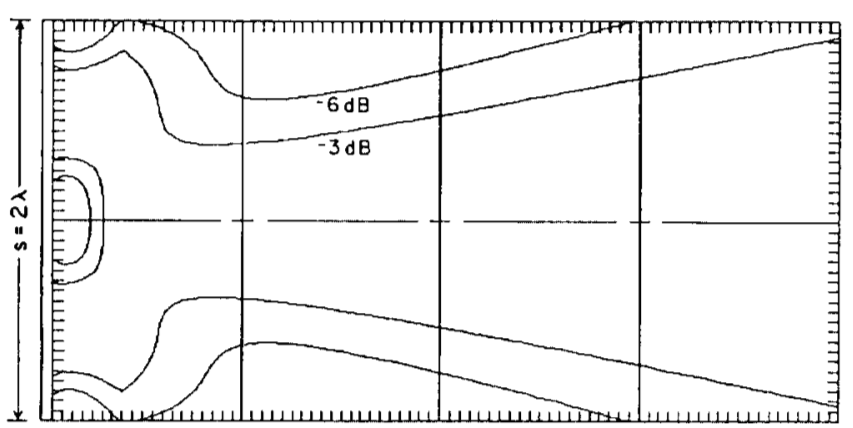

(a)

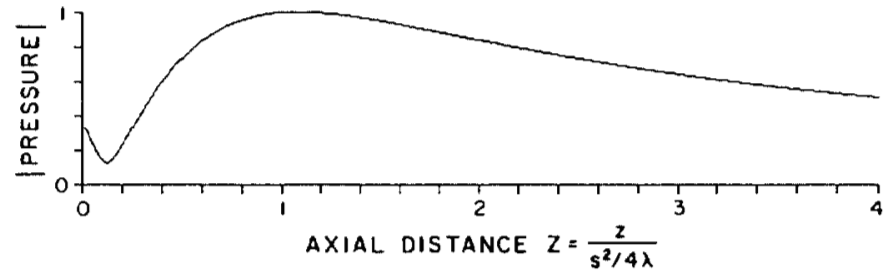

(b)

Fig. 4. Square source with sides equal to $2 \lambda$. (a) Sound pressure contour normalized at each axial distance. (b) Normalized magnitude of on-axis pressure.

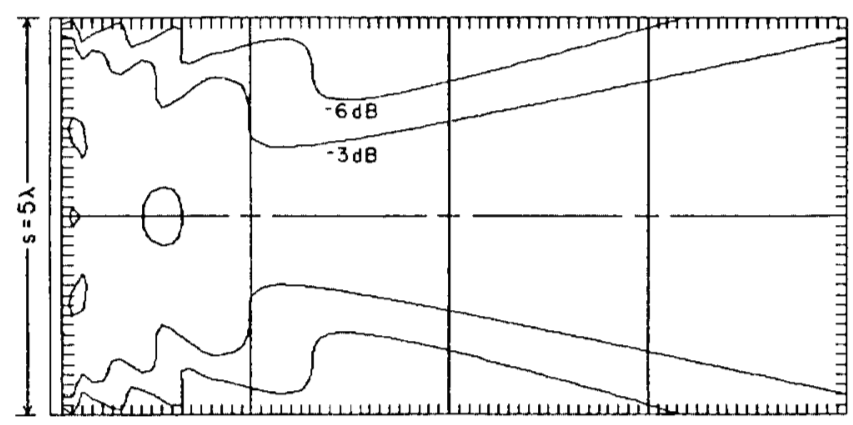

(a)

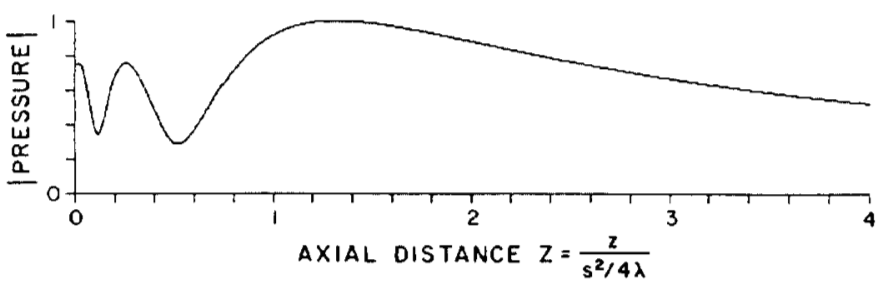

(b)

Fig. 5. Square source with sides equal to $5 \lambda$. (a) Sound pressure contour normalized at each axial distance. (b) Normalized magnitude of on-axis pressure.

of a circular source as noted by Lockwood and Willette [2]. Whereas Lockwood and Willette [2] showed the the field for only one rectangular source, comparisons are made here for a range of sizes of circular and square sources. These results show that similarities exist in the beam widths and locations of on-axis minima for similarly sized circular and square sources. Also, as the size of the source becomes large compared to a wavelength, the nor-

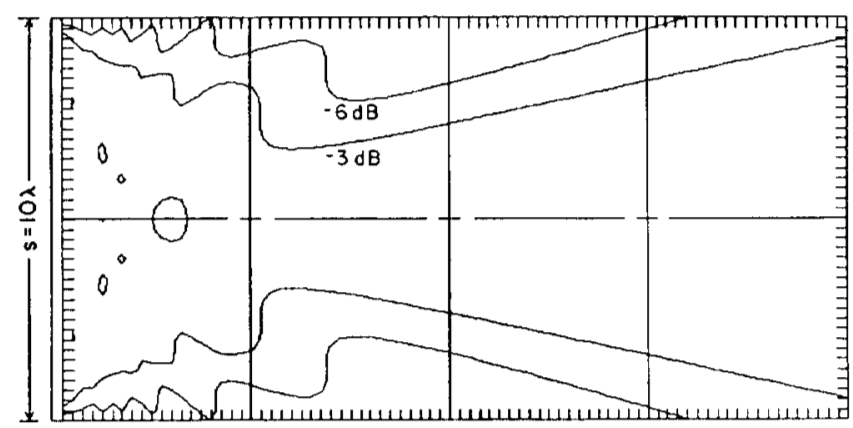

(a)

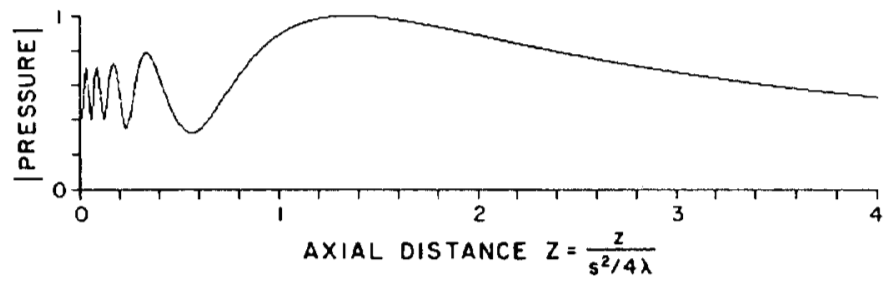

(b)

Fig. 6. Square source with sides equal to $10 \lambda$. (a) Sound pressure contour normalized at each axial distance. (b) Normalized magnitude of on-axis pressure.

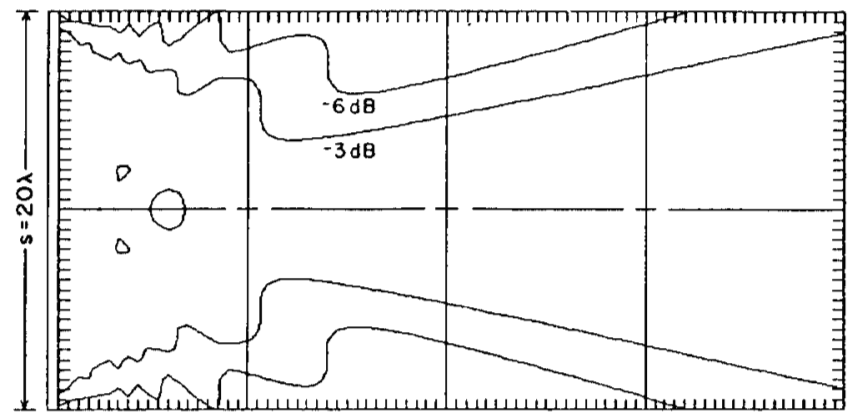

(a)

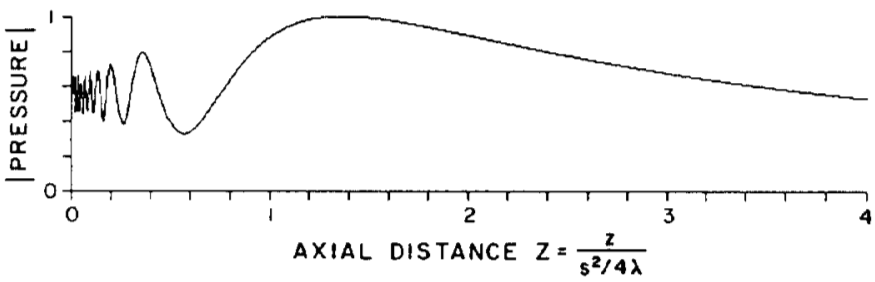

(b)

Fig. 7. Square source with sides equal to $20 \lambda$. (a) Sound pressure contour normalized at each axial distance. (b) Normalized magnitude of on axis pressure.

malized field no longer changes except very near the source (see for example Figs. 7 and 8 ).

The field for a rectangular source of dimensions suitable for a linear phased array element, with sides $0.05 \lambda$ $\left(s_{1}\right)$ by $10 \lambda\left(s_{2}\right)$, is shown for two perpendicular sections in Figs. 11 and 12. Note that the two plots use their respective values of $s$ for axial distance normalization, i.e., $s_{1}$ and $s_{2}$ for Figs. 11 and 12, respectively. Thus, the axial 


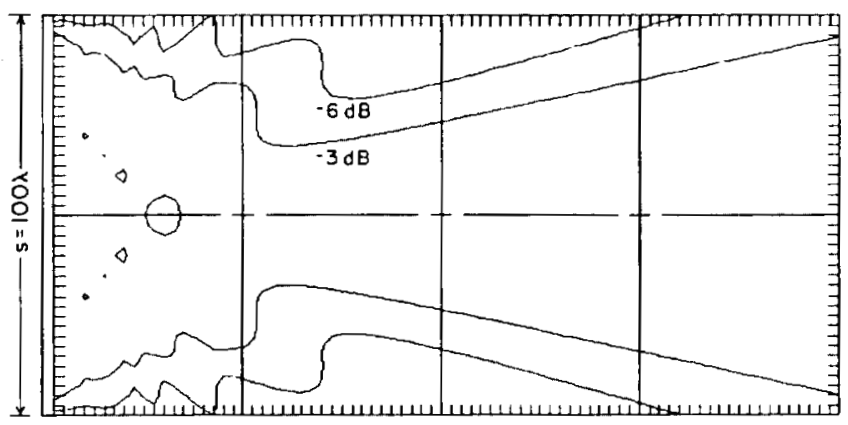

(a)

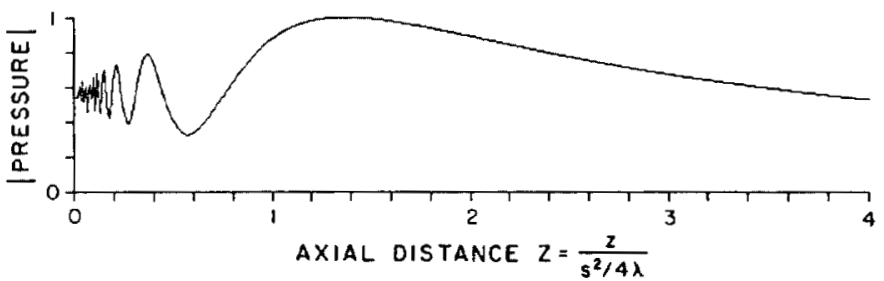

(b)

Fig. 8. Square source with sides equal to $100 \lambda$. (a) Sound pressure contour normalized at each axial distance. (b) Normalized magnitude of onaxis pressure.

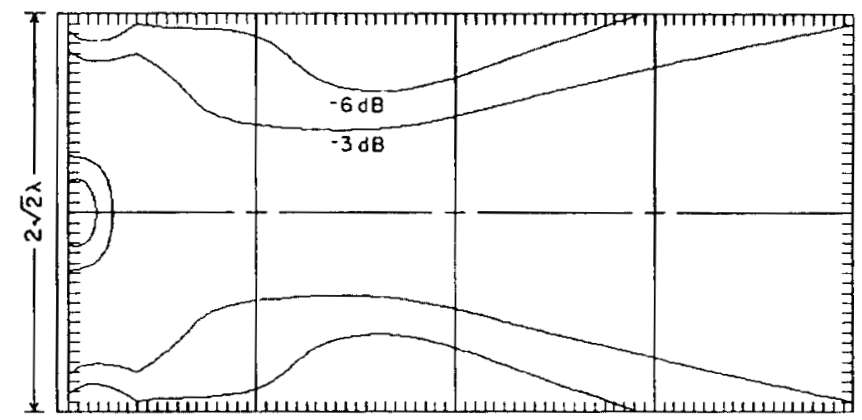

Fig. 9. Square source with sides equal to $2 \lambda$. Sound pressure contour normalized at each axial distance for section with $x=y$ (see Fig. 1).

fields shown in Figs. 11(b) and 12(b) are actually the same: the axial distances in each figure are relative to different nearfield-farfield transition distances. In fact, Marini and Rivenez [3] have divided axial field plots for rectangular sources into three zones: the very near field (near field for both dimensions), the near field (near field for the longer dimension) and the far field (far field for both dimensions). The similarities of the field sections for this rectangular source with those for equivalently sized square sources, Figs. 2 and 6, respectively, demonstrate the near independence of the longitudinal field patterns parallel to each side of a rectangular source. Accordingly, the length of a rectangular source in one direction, e.g., $x$, has little effect on the beam width produced in the other direction, $y$, of the source.

The effects of attenuation were examined by calculating the field for a square source, with sides of 10 wavelengths, radiating into media with attenuation coefficients
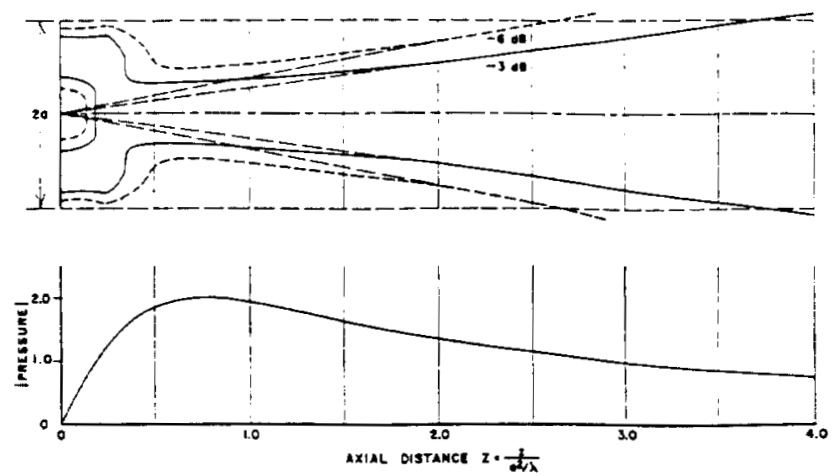

(a)
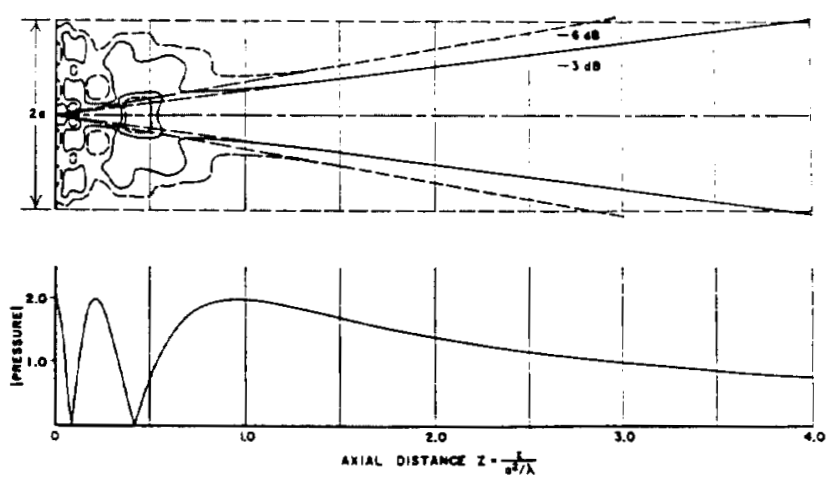

(b)
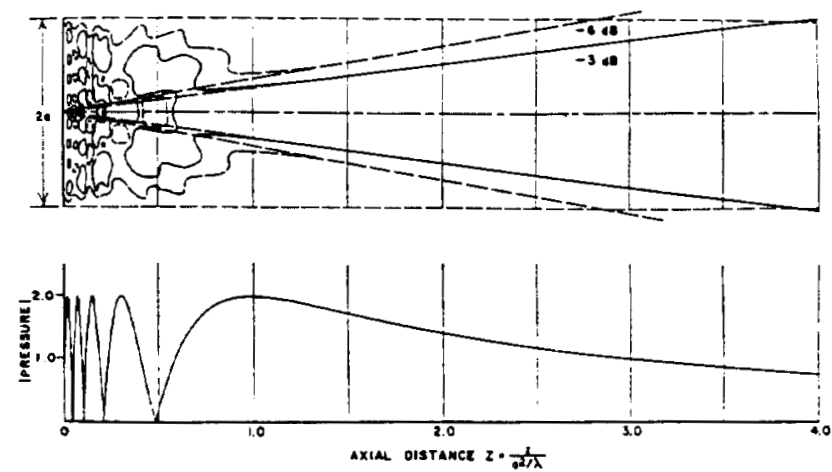

(c)

Fig. 10. Sound pressure contours normalized at each axial distance and normalized magnitudes of on-axis pressure for circular sources of diameters [1, Figs. 7-9]. (a) $2.0 \lambda$. (b) $5.0 \lambda$. (c) $10.0 \lambda$.

of $0.04,0.08$ and $0.12 \mathrm{~cm}^{-1}$ at $1 \mathrm{MHz}$ (see Fig. 13). To eliminate frequency dependence, the attenuation was incorporated as a constant value of attenuation per wavelength, $\alpha \lambda$. The values of $\alpha \lambda$ corresponding to the attenuation coefficients given above are computed to be 0.006 , 0.012 and 0.018 , respectively, using a value of $1.5 \times 10^{5}$ $\mathrm{cm} / \mathrm{s}$ for the speed of sound. Fig. 13(a) shows the contour plot, normalized at each axial distance, for the highest attenuation, $\alpha \lambda=0.018$. The normalized contour plot of the field varied little with attenuation as can be readily seen by comparing Fig. 13(a) with Fig. 6(a), which shows the field for the same source radiating into a lossless medium. This result is interesting because it demonstrates that the attenuation has little effect on the normalized field 


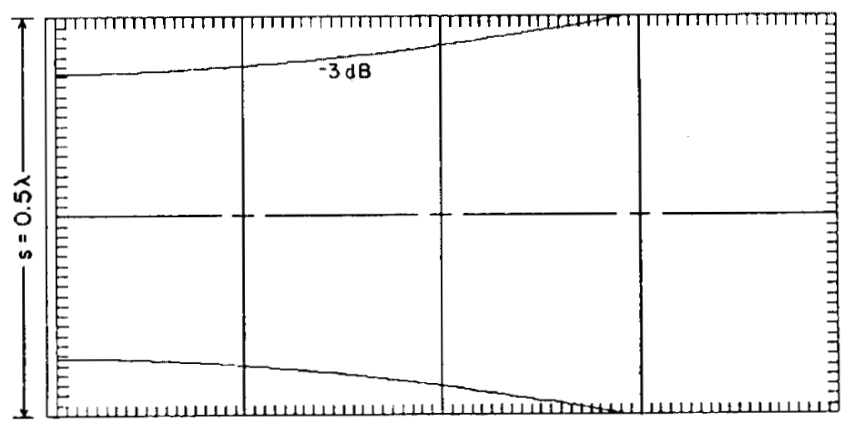

(a)

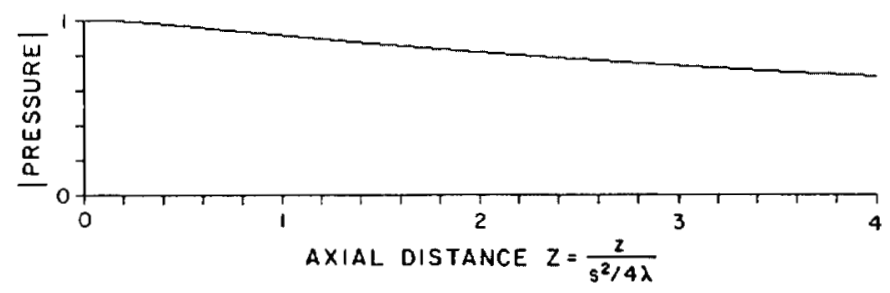

(b)

Fig. 11. Rectangular source with sides equal to $0.5 \lambda$ and $10.0 \lambda$. Field shown is in plane of the $0.5-\lambda$ length with $z$ distances relative to nearfield-farfield distance for square source with sides of length $0.5 \lambda$. (a) Sound pressure contour normalized at each axial distance (b) Normalized magnitude of on-axis pressure.

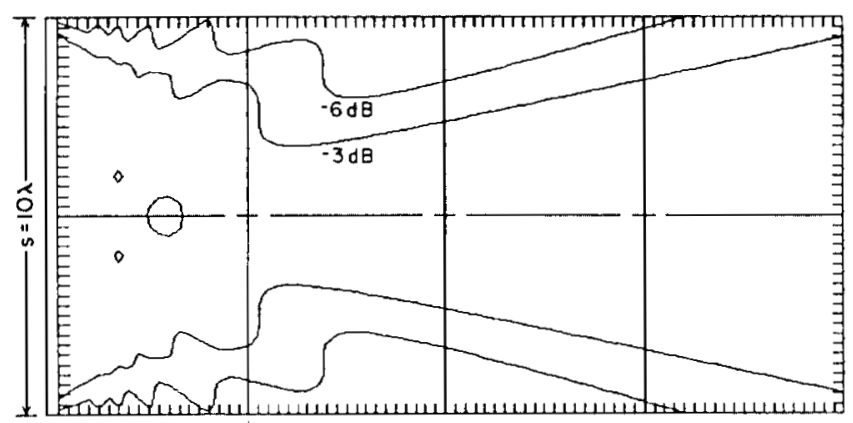

(a)

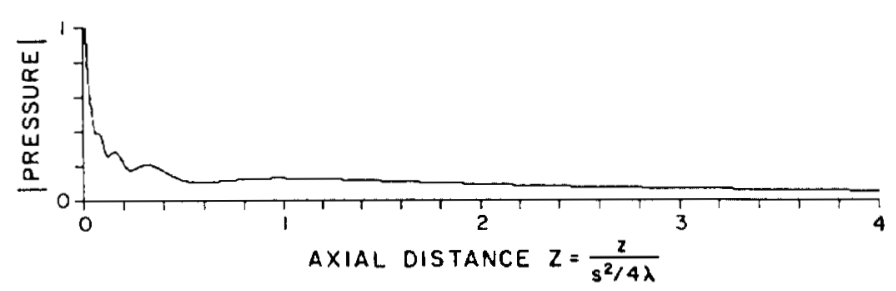

(b)

Fig. 12. Rectangular source with sides equal to $0.5 \lambda$ and $10.0 \lambda$. Field shown is in plane of $10.0-\lambda$ length with $z$ distances relative to nearfieldfarfield distance for square source with sides of length $10 \lambda$. (a) Sound pressure contour normalized at each axial distance. (b) Normalized magnitude of on-axis pressure.

transverse to the direction of propagation. On the other hand, Fig. 13(b) shows the substantial effect of attenuation on the axial pressure field, as expected. In addition to the decreased amplitude with distance from the source,

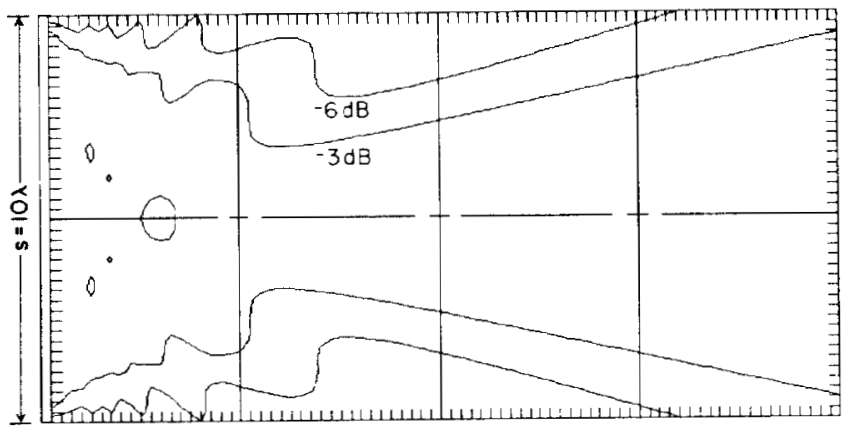

(a)

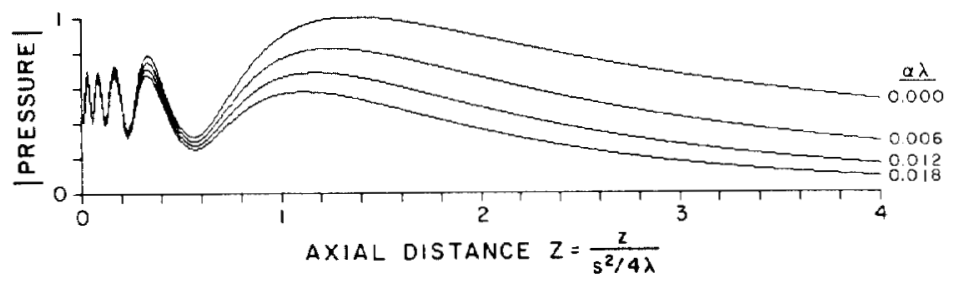

(b)

Fig. 13. Square source with sides equal to $10 \lambda$. (a) Sound pressure contour normalized at each axial distance in a medium, with $\alpha \lambda=0.018$. (b) Magnitude of on-axis pressure for $\alpha \lambda=0,0.006,0.012$, and 0.018 normalized to lossless case, $\alpha \lambda=0$.

the last axial maximum is shifted significantly closer to the source with increasing attenuation.

\section{CONCLUSION}

An accurate and efficient method was developed for determining the field of a plane source, which can be divided into rectangular elements, surrounded by a rigid baffle. The method sums the contribution to the pressure at a point from rectangular elements chosen to be small enough that the far field approximation is appropriate at the field point.

Though limited comparisons have been made in the past [2], [3], [5], this study compared the fields from square sources and those from circular sources for a range of sizes. In general, the fields are similar, but the field from a square source exhibited a more uniform pattern in the near field and has no nulls on axis. Further, for a rectangular source, the longitudinal section field profiles in the two planes parallel to the sides are largely independent. Radiation into an attenuating medium had little effect on the normalized transverse fields, but modified substantially the axial field.

\section{REFERENCES}

[1] J. Zemanek, "Beam behavior within the nearfield of a vibrating piston," J. Acoust. Soc. Am., vol. 49, no. 1, pp. 18i-191, Jan. 1971.

[2] J. C. Lockwood and J. G. Willette, "High-speed method for computing the exact solution for the pressure variations in the nearfield of a baffled piston," J. Acoust. Soc. Am. vol. 53, no. 3. pp. 735-741, Mar. 1973

[3] J. Marini and J. Rivenez, "Acoustic fields from rectangular ultrasonic transducers for nondestructive testing and medical diagnosis." Uttrason.. vol. 12, pp. 251-256. Nov. 1974. 
[4] D. A. Hutchins, H. D. Mair. P. A. Puhach, and A. J. Osei, "Continuous-wave pressure fields of ultrasonic transducers," J. Acoust. Soc. Am., vol. 80, no. 1, pp. 1-12, July 1986

[5] A. Weyns, "Radiation field calculations of pulsed ultrasonic transducers. Part 1: Planar circular, square and annular transducers," $U t$ trason., vol. 18 , pp. 183-188. July 1980.

[6] D. A. Hutchins, H. D. Mair, and R. G. Taylor, "Transient pressure fields of PVDF transducers," J. Acoust. Soc. Am., vol. 82, no. 1, pp. 183-192, July 1987

[7] A. Freedman, "Sound field of a rectangular piston," J. Acoust. Soc. Am., vol. 32, no. 2, pp. 197-209, Feb. 1960

[8] P. R. Stepanishen, "The time-dependent force and radiation impedance on a piston in a rigid infinite planar baffle," J. Acoust. Soc. Am., vol. 49, no. 3, pp. 841-849, Mar. 1971.

[9] K. B. Ocheltree, "Theoretical analysis of ultrasonic linear phased arrays for hyperthermic treatment," M.S. thesis, Dept. Elec. and Comp. Eng., Univ. of Illinois, Urbana, IL, 1984.

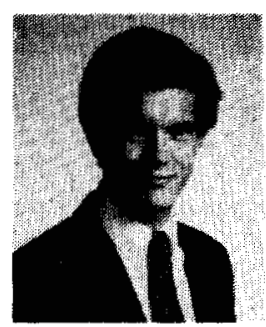

Kenneth B. Ocheltree ( $\left.S^{\prime} 82-M^{\prime} 82-S^{\prime} 82-M^{\prime} 86\right)$ was born on June 8,1960 , in Norfolk, VA. He received the B.S. degree in electrical engineering from Virginia Polytechnic Institute, Blacksburg, VA, in 1982, and the M.S. and Ph.D. degrees in electrical engineering from the University of Illinois at Urbana-Champaign in 1984 and 1987, respectively. While at the University of Illinois, he was involved in research on ultrasound hyperthermia.

He has been with the IBM T.J. Watson Re- search Center, Yorktown Heights, NY since 1987. His current research activities involve high speed local area networks and network management.

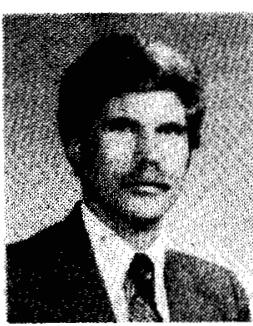

Leon A. Frizzell (S'71-M'74-SM'82) was born in West Stewartstown, NH, on September 12, 1947. He received the B.S. degree in physics from the University of New Hamsphire, Durham, NH in 1969, and the M.S. and Ph.D. degrees in elec trical engineering from the University of Rochester, Rochester, NY, in 1971 and 1976, respectively.

Since 1975 , he has been in the Department of Electrical and Computer Engineering at the University of Illinois at Urbana-Champaign, where he is currently an Associate Professor of Electrical and Computer Engineering and of Bioengineering. His research interests include ultrasonic tissue characterization, ultrasonic biological effects, hyperthermia, and ultrasonic bioengineering.

Dr. Frizzell is a member of the Acoustical Society of America, the American Association for the Advancement of Science, Sigma Xi, and is a Fellow of the American Institute of Ultrasound in Medicine. 